
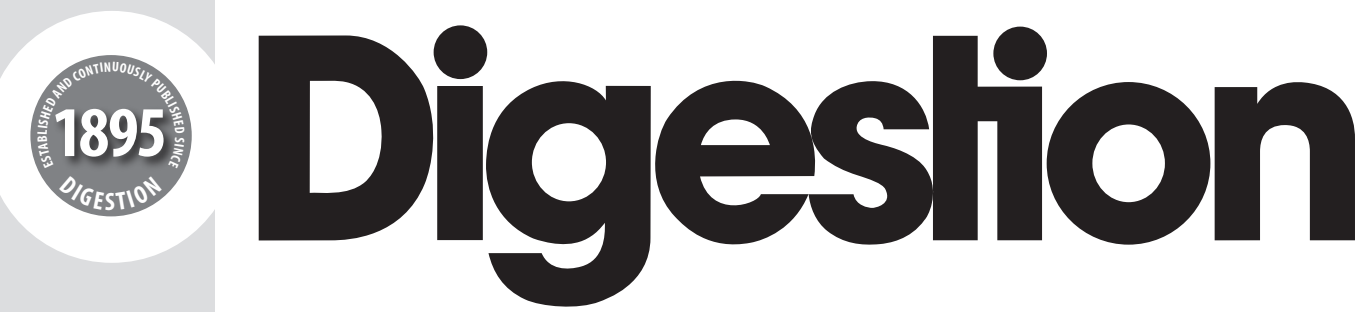

\title{
International Journal of Gastroenterology
}

Founded as "Archiv für Verdauungskrankheiten" 1895 by I. Boas

Continued as "Gastroenterologia"1939-1967

Former Editors: P. Morawitz (1934-1936), R. Staehelin (1937-1943), A. Hurst (1940-1945), W. Löffler (1943-1961), T.C. Hunt (1947-1967), N. Henning (1953-1962), B. Ihre (1953-1967),

H. Bartelheimer (1963-1967), M. Demole (1963-1971), H. Kapp (1968-1970),

R. Lambert (1972-1978), W. Creutzfeldt (1979-1992), R. Arnold (1993-2003),

C. Beglinger (2004-2011), B. Göke (2004-2014), Y. Shinomura (2012-2015)

\section{Editors-in-Chief}

V. Ellenrieder, Göttingen

K. Higuchi, Osaka

\section{Managing Editors}

S. Cameron, Göttingen

U. König, Göttingen

\section{Associate Editors}

A. Andoh, Otsu

H. Baba, Kumamoto

A. Canbay, Magdeburg

T. Gotoda, Tokyo

H. Isomoto, Yonago

R. Jakobs, Ludwigshafen

H. Kashida, Osaka

A.O. König, Göttingen

N. Malek, Tübingen

P.J. Michl, Halle (Saale)

Y. Naito, Kyoto

A. Nakajima, Yokohama

G. Rogler, Zurich

Y. Sasaki, Kumamoto

A. Shiotani, Kurashiki

M. Tanaka, Fukuoka

A. Vogel, Hannover

H. Yamamoto, Kawasaki

\section{Editorial Board}

A. Amanzada, Göttingen

W. Fischbach, Aschaffenburg

J. Gaedcke, Göttingen

G. Holtmann, Woolloongabba, Qld.

F. Itoh, Kawasaki

F. Kolligs, Berlin

F. Lordick, Leipzig

M.P. Lutz, Saarbrücken

H. Matsubara, Chiba

D. Müller, Marburg

M. Nakamura, Tokyo

A. Neesse, Göttingen

M. Pavel, Berlin

A. Perren, Bern

R.R. Plentz, Tübingen

J. Rosendahl, Leipzig

T. Seufferlein, Ulm

T. Sugai, Morioka

A. Weber, Munich

Y. Kinoshita, Izumo 
S. Karger

Medical and Scientific Publishers

Basel $\cdot$ Freiburg $\cdot$ Hartford $•$ Oxford $•$ Bangkok $\cdot$ Dubai $\cdot$ Kuala Lumpur $\cdot$ Melbourne $\cdot$ Mexico City $\cdot$ Moscow $•$ New Delhi $\bullet$ Paris $\bullet$ Shanghai $\bullet$ Tokyo

\section{Disclaimer}

The statements, opinions and data contained in this publication are solely those of the individual authors and contributors and not of the publisher and the editor(s). The appearance of advertisements in the journal is not a warranty, endorsement, or approval of the products or services advertised or of their effectiveness, quality or safety. The publisher and the editor(s) disclaim responsibility for any injury to persons or property resulting from any ideas, methods, instructions or products referred to in the content or advertisements.

Drug Dosage

The authors and the publisher have exerted every effort to en sure that drug selection and dosage set forth in this text are in accord with current recommendations and practice at the time of publication. However, in view of ongoing research, changes in government regulations, and the constant flow of information relating to drug therapy and drug reactions, the reader is urged to check the package insert for each drug for any change in indications and dosage and for added warnings and precautions. This is particularly important when the recommended agent is a new and/or infrequently employed drug.
All rights reserved.

No part of this publication may be translated into other languages, reproduced or utilized in any form or by any means, electronic or mechanical, including photocopying, recording, microcopying, or by any information storage and retrieval system, without permission in writing from the publisher or, in the case of photocopying, direct payment of a specified fee to the Copyright Clearance Center (see "General Information")

(c) Copyright 2018 by S. Karger AG

CH-4009 Basel (Switzerland)

Printed on acid-free and non-aging paper (ISO 9706)

\section{KARGER}


No. 1

Review

1 Endoscopic Ultrasound Guided Choledocho-Enterostomy by Using Lumen Apposing Metal Stent in Patients with Failed Endoscopic Retrograde Cholangiopancreatography: A Literature Review

Jain, D. (Philadelphia, PA); Shah, M.; Patel, U.; Sharma, A.; Singhal, S. (Houston, TX)

Original Papers

11 A Web-Based Education Program for Colorectal Lesion Diagnosis with Narrow Band Imaging Classification Aihara, H.; Kumar, N.; Thompson, C.C. (Boston, MA)

19 Mucosal Two-Step Pathogenesis in Gastroesophageal Reflux Disease: Repeated Weakly Acidic Stimulation and Activation of Protease-Activated Receptor-2 on Mucosal Interleukin-8 Secretion

Winkelsett, L.; Malfertheiner, P.; Wex, T. (Magdeburg); Kandulski, A. (Magdeburg/Regensburg)

26 Long-Term Outcome of Endoscopic Balloon Dilatation for Strictures in Patients with Crohn's Disease Taida, T.; Nakagawa, T.; Ohta, Y.; Hamanaka, S.; Okimoto, K.; Saito, K.; Maruoka, D.; Matsumura, T.; Arai, M.; Katsuno, T.; Kato, N. (Chiba)

33 Predictive Factors of Portal Hypertensive Enteropathy Exacerbation in Patients with Liver Cirrhosis: A Capsule Endoscopy Study

Kunihara, S.; Oka, S.; Tanaka, S.; Otani, I.; Igawa, A.; Nagaoki, Y.; Aikata, H.; Chayama, K. (Hiroshima)

41 Initial Management of Colonic Diverticular Bleeding: Observational Study

Wada, M.; Kato, M.; Hirai, Y.; Kubosawa, Y.; Sunata, Y.; Abe, K.; Hirata, T.; Takada, Y.; Banno, S.; Takatori, Y.; Kinoshita, S.; Mori, H.; Takabayashi, K.; Kikuchi, M.; Kikuchi, M.; Suzuki, M.; Kanai, T.; Uraoka, T. (Tokyo)

48 Clinical Significance of Reddish Depressed Lesions Observed in the Gastric Mucosa after Helicobacter pylori Eradication Kotachi, T.; Ito, M.; Boda, T.; Kiso, M.; Masuda, K.; Hata, K.; Kawamura, T. Sanomura, Y. (Hiroshima); Yoshihara, M. (Higashihiroshima); Tanaka, S.; Chayama, K. (Hiroshima)

56 Modulation of Pacemaker Potentials in Murine Small Intestinal Interstitial Cells of Cajal by Gamisoyo-San, a Traditional Chinese Herbal Medicine

Kim, D.; Kim, J.N. (Yangsan); Nam, J.H. (Yangsan/Kyungju); Lee, J.R.; Kim, S.C. (Gyeongsan); Kim, S.C. (Yangsan)

\section{No. 2}

Short Communication

69 Combined Systemic Chemotherapy and CT-Guided High-Dose-Rate Brachytherapy for Isolated Local Manifestation of Pancreatic Cancer after Surgical Resection Franck, C.; Hass, P.; Malfertheiner, P. (Magdeburg); Ricke, J.; Seidensticker, M. (Magdeburg/Munich); Venerito, M. (Magdeburg)

Original Papers

75 Diagnostic Accuracy of Latex Agglutination Turbidimetric Immunoassay in Screening Adolescents for Helicobacter pylori Infection in Japan

Tsutsumi, K. (Oita/Akita); Kusano, C.; Suzuki, S.; Gotoda, T. (Tokyo); Murakami, K. (Oita)

81 Usefulness of Fecoflowgram for Assessment of Defecation after Intersphincteric Resection

Kawahara, H.; Mouri, T.; Ishida, K.; Matsumoto, N.; Akiba, T. (Chiba); Yanaga, K. (Tokyo)

87 The Concentration of lodine in Perigastric Adipose Tissue: A Novel Index for the Assessment of Serosal Invasion in Patients with Gastric Cancer after Neoadjuvant Chemotherapy

Yang, L.; Li, Y.; Shi, G.; Zhou, T.; Tan, B. (Shijiazhuang)

95 Mucosally Expressed Cytokines Are Associated with the Esophageal Motility Function

Fukaura, K.; Ihara, E.; Ogino, H.; Iboshi, Y.; Muta, K.; Xiaopeng, B. Hamada, S.; Hata, Y.; Iwasa, T.; Aso, A.; Nakamura, K.; Ogawa, Y. (Fukuoka)

104 Corticosteroid Therapy Improves the Outcome of Autoimmune Hepatitis-Induced Acute Liver Failure Anastasiou, O.E.; Dogan-Cavus, B. (Essen); Kucukoglu, O. (Magdeburg); Baba, H.; Kahraman, A.; Gerken, G. (Essen); Schramm, C. (Hamburg); Canbay, A. (Magdeburg)

112 Endoscopic Ultrasound Features of Multiple Endocrine Neoplasia Type 1-Related versus Sporadic Pancreatic Neuroendocrine Tumors: A Single-Center Retrospective Study Tamagno, G. (Marburg/Dublin); Scherer, V. (Marburg); Caimo, A. (Dublin); Bergmann, S.R.; Kann, P.H. (Marburg)

119 Comparison of Small Bowel Lesions Using Capsule Endoscopy in Ulcerative Colitis and Crohn's Disease: A Single-Center Retrospective Analysis

Ninomiya, K.; Hisabe, T.; Okado, Y.; Takada, Y.; Yamaoka, R.; Sato, Y.; Kishi, M.; Takatsu, N.; Matsui, T.; Ueki, T.; Yao, K.; Hirai, F. (Fukuoka)

127 Characterization of Helicobacter pylori-Naïve Early Gastric Cancers

Yamada, A.; Kaise, M.; Inoshita, N.; Toba, T.; Nomura, K.; Kuribayashi, Y.; Yamashita, S.; Furuhata, T.; Kikuchi, D.; Matsui, A.; Mitani, T.; Ogawa, O.; Iizuka, T.; Hoteya, S. (Tokyo)

\section{KARGER}

E-Mail karger@karger.com www.karger.com
(C) 2018 S. Karger AG, Basel

Access to full text and tables of contents, including tentative ones for forthcoming issues: www.karger.com/dig_issues 
No. 3

To the Point Article

135 Treatment Perspectives in Crohn's Disease

Vetter, M.; Neurath, M.F. (Erlangen)

Original Papers

143 Advanced Mucinous Colorectal Cancer: Epidemiology, Prognosis and Efficacy of Chemotherapeutic Treatment

Ott, C.; Gerken, M.; Hirsch, D.; Fest, P.; Fichtner-Feigl, S.; Munker, S. Schnoy, E.; Stroszczynski, C.; Vogelhuber, M.; Herr, W.; Evert, M.; Reng, M.; Schlitt, H.J.; Klinkhammer-Schalke, M. (Regensburg); Teufel, A. (Mannheim)

153 Esophageal Motility after Extensive Circumferential Endoscopic Submucosal Dissection for Superficial Esophageal Cancer

Kuribayashi, Y.; Iizuka, T.; Nomura, K.; Furuhata, T.; Yamashita, S.; Matsui, A.; Kikuchi, D.; Mitani, T.; Kaise, M.; Hoteya, S. (Tokyo)

161 The Role of an Undifferentiated Component in Submucosal Invasion and Submucosal Invasion Depth After Endoscopic Submucosal Dissection for Early Gastric Cancer

Miyahara, K. (Hiroshima); Hatta, W. (Sendai); Nakagawa, M. (Hiroshima); Oyama, T. (Saku); Kawata, N. (Shizuoka); Takahashi, A. (Saku); Yoshifuku, Y. (Hiroshima); Hoteya, S. (Tokyo); Hirano, M. (Joetsu); Esaki, M. (Kitakyushu); Matsuda, M. (Toyama); Ohnita, K. (Nagasaki); Shimoda, R. (Saga); Yoshida, M. (Kashihara); Dohi, O. (Kyoto); Takada, J. (Gifu); Tanaka, K. (Matsumoto); Yamada, S. (Kyoto); Tsuji, T. (Akita); Ito, H. (Osaki); Aoyagi, H. (Fukui); Shimosegawa, T. (Sendai)

169 Temporal Trends in Helicobacter pylori Eradication Success in a Test-and-Treat Population

Boltin, D.; Schmilovitz-Weiss, H.; Gingold-Belfer, R.; Leibovitzh, H.; Snir, Y.; Perets, T.T.; Dickman, R.; Levi, Z.; Niv, Y. (Petah Tikva)

175 Usefulness of Demarcation of Differentiated-Type Early Gastric Cancers after Helicobacter pylori Eradication by Magnifying Endoscopy with Narrow-Band Imaging Akazawa, Y.; Ueyama, H.; Yao, T.; Komori, H.; Takeda, T.; Matsumoto, K.; Matsumoto, K.; Asaoka, D.; Hojo, M.; Watanabe, S.; Nagahara, A. (Tokyo)

185 Factors Associated with Gastric Black Spot, White Flat Elevated Mucosa, and Cobblestone-Like Mucosa: A Cross-Sectional Study

Hatano, Y. (Shobara); Haruma, K. (Okayama); Kamada, T.; Shiotani, A. (Kurashiki); Takahari, K. (Onomichi); Matsumoto, M. (Minami-ku); Uchida, O. (Wakaba-ku)

194 Pathogenesis of Potassium-Competitive Acid Blocker-Resistant Non-Erosive Reflux Disease Kawami, N.; Hoshino, S.; Hoshikawa, Y.; Takenouchi, N.; Umezawa, M.; Hanada, Y.; Kaise, M.; Iwakiri, K. (Tokyo)

No. 4

Original Papers

201 Gastroesophageal Reflux Disease-Related Disorders of Systemic Sclerosis Based on the Analysis of 66 Patients Matsuda, R.; Yamamichi, N. (Tokyo); Shimamoto, T. (Tokyo/Chiba); Sumida, H.; Takahashi, Y.; Minatsuki, C.; Kodashima, S.; Ono, S.; Niimi, K.; Tsuji, Y.; Sakaguchi, Y.; Saito, I.; Kataoka, Y.; Asada-Hirayama, I.; Kakimoto, H.; Yakabi, S.; Takeuchi, C.; Matsumoto, Y.; Tamaki, Z.; Fujishiro, M.; Asano, Y.; Sato, S.; Koike, K. (Tokyo)
209 Predictive Factors for Differentiating Pancreatic Cancer-Associated Diabetes Mellitus from Common Type 2 Diabetes Mellitus for the Early Detection of Pancreatic Cancer Dong, X. (Hangzhou/Yiwu); Lou, Y.B.; Mu, Y.C. (Yiwu); Kang, M.X.; Wu, Y.L. (Hangzhou)

217 Influence of Simethicone Added to the Rinse Water during Colonoscopies on Polyp Detection Rates: Results of an Unintended Cohort Study

Kutyla, M.; O'Connor, S.; Gurusamy, S.R.; Gururatsakul, M.; Gould, K.; Whaley, A.; Kendall, B.J.; Hourigan, L.; Holtmann, G.J. (Brisbane, QLD)

222 Lavender Color in Linked Color Imaging Enables Noninvasive Detection of Gastric Intestinal Metaplasia

Ono, S.; Kato, M.; Tsuda, M.; Miyamoto, S.; Abiko, S.; Shimizu, Y.; Sakamoto, N. (Sapporo)

231 Effects of Gamisoyo-San Decoction, a Traditional Chinese Medicine, on Gastrointestinal Motility

Shin, S.J.; Kim, D.; Kim, J.S.; Kim, I. (Yangsan); Lee, J.R.; Kim, S.C. (Gyeongsan); Kim, B.J. (Yangsan)

238 Significance of the Lysyl Oxidase Members Lysyl Oxidase Like 1, 3, and 4 in Gastric Cancer

Kasashima, H.; Yashiro, M.; Okuno, T.; Miki, Y.; Kitayama, K.; Masuda, G.; Kinoshita, H.; Morisaki, T.; Fukuoka, T.; Hasegawa, T.; Sakurai, K.; Toyokawa, T.; Kubo, N.; Tanaka, H.; Muguruma, K.; Hirakawa, K.; Ohira, M. (Osaka)

249 Magnifying Endoscopy with Narrow Band Imaging in Estimating the Invasion Depth of Superficial Esophageal Squamous Cell Carcinomas

Mizumoto, T. (Hiroshima); Hiyama, T. (Higashihiroshima); Quach, D.T (Hochiminh); Sanomura, Y.; Urabe, Y.; Oka, S.; Arihiro, K.; Tanaka, S.; Chayama, K. (Hiroshima)

257 Association between Higher Body Mass Index and PouchRelated Complications during Restorative Proctocolectomy in Patients with Ulcerative Colitis

Horio, Y.; Uchino, M.; Bando, T.; Chohno, T.; Takesue, Y.; Ikeuchi, H. (Nishinomiya)

263 Anti-EGF Receptor-Based Conversion Chemotherapy in RAS Wild-Type Colorectal Cancer Patients: Impact on Survival and Resection Rates

Koch, C.; Schmidt, N.; Winkelmann, R.; Eichler, K.; Pession, U.;

Bechstein, W.O.; Zeuzem, S.; Waidmann, O.; Trojan, J. (Frankfurt am Main)

Short Communication

270 A Pilot Trial to Quantify Plasma Exosomes in Colorectal Cancer Screening from the International Collaborative Study between Chile and Japan

Kobayashi, M.; Kawachi, H.; Hurtado, C.; Wielandt, A.M.; Ponce, A. (Santiago); Karelovic, S.; Pasternak, S.; Delgado, C.; Pinto, P.; Carrasco, H. (Punta Arenas); Ito, T.; Okada, T.; Tanaka, K.; Odagaki, T.; Zárate, A.J.; Kronberg, U.; López-Köstner, F.; Tsubaki, M. (Santiago); Kawano, T.; Eishi, Y. (Tokyo)

275 Acknowledgment to Referees 